\title{
Mechanisms of 5'-Inosinic Acid Accumulation by Permeability Mutants of Brevibacterium ammoniagenes. I. Genetical Improvement of 5'-IMP Productivity of a Permeability Mutant of B. ammoniagenes ${ }^{\dagger}$
}

\author{
Sadao TeShIBA and Akira FuruyA \\ Tokyo Research Laboratories, Kyowa Hakko Kogyo Co. Ltd., \\ Machıda-shı, Tokyo 194, Japan
}

Received March 5, 1982

\begin{abstract}
In order to establish industrial production of $5^{\prime}$-nosinic acid (5'-IMP), a permeablity mutant, KY13171, of Brevibacterium ammoniagenes, which accumulated 7 to 8 grams of $5^{\prime}$-IMP per liter and 4 to 6 grams of hypoxanthine ( $\mathrm{Hx}$ ) per liter (calculated as $5^{\prime}$-IMP), was improved by a genetical procedure. Further improved mutants were selected stepwise through repeating mutational work. The finally selected mutant. KY13369, accumulated 20 to 27 grams of 5'-IMP per liter, but not Hx.

Increased productivity of $5^{\prime}$-IMP and decreased productivity of $\mathrm{Hx}$ were not caused by the changes in 5'-IMP degrading activity, because these actıvities were not significantly different among the mutants. These results appear to indicate that the increased accumulation of 5 -IMP may be caused by the improvement in membrane permeability for 5'-IMP. However, the changes in phospholipid and fatty acid compositions were not enough to explain the increased permeability.
\end{abstract}

Since the discovery of flavour enhancing activities of $5^{\prime}$-inosinic acid (5'-IMP) and 5'guanylic acid by Kuninaka, strenuous efforts have been made for establishment of industrial production of these nucleotides. During our studies on direct production of 5'-IMP by mutants of Brevibacterium ammoniagenes, it was found that an adenine-leaky auxotrophic mutant, KY13102, produced considerable amounts of 5 -IMP in the culture broth-the maximum accumulation was 12.4 grams per liter. ${ }^{1)}$ However, 5'-IMP accumulation by KY13102 was severely affected by very low concentrations of manganese ions $\left(\mathrm{Mn}^{2+}\right)$ and excessive amounts of $\mathrm{Mn}^{2+}$ (more than $20 \mu \mathrm{g}$ per liter) drastically inhibited the 5'-IMP accumulation, making it difficult to carry out practical production with the mutant. Through our work on mechanisms of the $\mathrm{Mn}^{2+}$ action, it has been found that excessive amounts of the ion repress membrane permeability for 5'-IMP and consequently excretion of $5^{\prime}$-IMP formed in the cells was inhibited, and hypoxanthine
$(\mathrm{Hx})$, a degradation product of $5^{\prime}$-IMP, was accumulated. $^{2)}$

For industrial production of $5^{\prime}$-IMP, it was desirable to obtain mutants in which accumulation of 5'-IMP was not affected by $\mathrm{Mn}^{2+}$ (manganese-insensitive type). A number of colonies were picked up from the survivors of KY13102 after mutagenic treatment and tested for their 5'-IMP productivities in the presence of excessive $\mathrm{Mn}^{2+}$, and finally such a type of mutant, KY13171, was obtained, which accumulated 7.5 grams of 5'-IMP per liter under excessive $\mathrm{Mn}^{2+}$ conditions. ${ }^{3)}$

For practical and economical production, the 5'-IMP productivity of KY13171 was not enough compared with that of KY13102. In order to improve KY13171, an attempt was made to release the feedback regulation in de novo purine biosynthesis by guanine nucleotides, ${ }^{4)}$ and a mutant, KY13184, which is devoid of IMP dehydrogenase and requirement of guanine for growth, was derived from KY13171. The mutant showed higher pro-

${ }^{\dagger}$ This is Part XLVIII of a series of reports entitled "Production of Nucleic Acid-related Substances by Fermentatıve Processes." 
ductivity than the parent, KY13171. All further improved mutants were derived from KY13184 through repeated cycles of mutation and selection. In the end, KY13369 was obtained, which accumulated 20 to 27 grams of 5'-IMP per liter. In this report, we will describe the isolation and characterization of these mutants and the results concerning the mechanisms of the increased 5'-IMP productivities of these mutants.

\section{MATERIALS AND METHODS}

Microorganism. All mutants were derived from Brevibacterium ammoniagenes KY3454 and are shown in Fig. 1. The 5'-IMP producing mutants, KY13102 and KY13171, were reported in our previous papers. ${ }^{1,3)}$ Isolation and characterization of the other mutants are described in this paper.

Media and cultivation. The preservation medium and the seed medium for cultures were the same as those reported in the previous paper. ${ }^{3)}$ The fermentation medium was composed of: glucose, $130 \mathrm{~g} ; \mathrm{KH}_{2} \mathrm{PO}_{4}, 10 \mathrm{~g} ; \mathrm{K}_{2} \mathrm{HPO}_{4}$, $10 \mathrm{~g} ; \mathrm{MgSO}_{3} \cdot 7$ aq., $10 \mathrm{~g} ; \mathrm{ZnSO}_{4} \cdot 7$ aq., $1 \mathrm{mg} ; \mathrm{Mn}^{2+}, 1 \mathrm{mg}$; cysteine $\cdot \mathrm{HCl}, \quad 20 \mathrm{mg} ; \quad$ thiamine $\cdot \mathrm{HCl}, \quad 5 \mathrm{mg} ; \quad \mathrm{Ca}-\mathrm{D}-$ pantothenate, $10 \mathrm{mg}$; nicotinate, $5 \mathrm{mg}$; biotin, $30 \mu \mathrm{g}$; corn steep liquor, $20 \mathrm{~g}$; adenine, $100 \mathrm{mg}$; guanine, $100 \mathrm{mg}$; and urea, $2 \mathrm{~g}$ (separately sterilized) in 1 liter of deionized water. The $\mathrm{pH}$ was adjusted to 8.3 with $5 \mathrm{~N} \mathrm{NaOH}$ before sterilization.

For accumulation of 5'-IMP, the organisms were cultivated as described previously with the following modifcations $^{3)}$ : On the first and second day of the culture, $0.2 \%$ of urea (final concentration) was added, and the $\mathrm{pH}$ of the culture broth was adjusted at $7.5 \sim 8.0$ with $50 \%$ ammonia water on the third day.

Assay of 5'-IMP and Hx. Determination of 5'-IMP and $\mathrm{Hx}$ in fermentation medium was performed by a paper chromatography method. ${ }^{5)}$ The amounts of 5'-IMP were expressed as 5'-IMP $\cdot \mathrm{Na}_{2} \cdot 7.5 \mathrm{H}_{2} \mathrm{O}$ and the amounts of $\mathrm{Hx}$ were calculated as $5^{\prime}$-IMP. The sum of accumulated $5^{\prime}$ IMP and calculated $5^{\prime}$-IMP from accumulated $\mathrm{Hx}$ is presented as total $5^{\prime}$-IMP.

Enzyme assays. Preparation of the cell extract used as enzyme source was as described previously. ${ }^{3}$ Alkaline phosphatase activity was determined by the $p$-nitrophenylphosphate method as described previously. ${ }^{3)}$ Assaying of $5^{\prime}$-IMP degrading activity was performed according to the method of Shiio ${ }^{6}$ with some modifications. The specific activity was estimated by measuring the liberated organic phosphate in the reaction mixture by the method of Chen. ${ }^{7)}$

Protein determination. Protein was determined by the method of Lowry et al. with crystalline bovine serum albumin as a standard.

Separation and identification of phospholipid. Cells cultivated in fermentation medium for 3 days at $30^{\circ} \mathrm{C}$ were harvested by centrifugation and then washed with $0.4 \mathrm{~N}$ $\mathrm{HCl}$ twice. The washed cells were lyophilized and extracted according to a modification of the method of Takinami et al $^{8)}$ Phospholipids were separated and analyzed by thinlayer chromatography with Merck silica gel G (Art. No. 5721). The silica gel was developed in three different solvent systems. The lipid components were characterized by their $R f$ values referring to those of authentic samples and their reactions with specific reagents.

Analysis of fatty acids. The cells cultivated in fermentation medium for 3 days at $30^{\circ} \mathrm{C}$ were separated and washed with cold $0.2 \mathrm{~N} \mathrm{HCl}$ once and $0.1 \mathrm{M}$ phosphate buffer ( $\mathrm{pH} 7.0)$ twice. Dried cells ( 0.4 grams) prepared by a freeze-drying procedure were hydrolyzed in $8 \mathrm{ml}$ of $4 \mathrm{~N}$ $\mathrm{HCl}$ with heating at $120 \sim 130^{\circ} \mathrm{C}$ for $2 \mathrm{hr}$ in a sealed tube. Fatty acids were extracted with ether three times and washed with distilled water, followed by dehydration with anhydrous sodium sulfate. Fatty acids thus obtained were converted to their methyl esters according to the procedure of Luddy et al. ${ }^{9)}$ which were then analyzed by gasliquid chromatography using a Perkin Elmer 900 type equipped with a flame ionization detector. A glass column $(2 \mathrm{~mm} \times 4$ feet $)$ packed with $15 \%$ DEGS coated with Chromosorb W was used. Operation conditions were as follows: The column temperature was raised from $100^{\circ} \mathrm{C}$ to $180^{\circ} \mathrm{C}$ at a rate of $4^{\circ} \mathrm{C}$ per minute; carrier gas, $\mathrm{He}$, and flow rate, $10 \mathrm{ml}$ per minute; fuel gas, $\mathrm{H}_{2}$, and flow rate, $15 \mathrm{ml}$ per minute. The individual fatty acids were characterized by comparison of their retention times with those of authentic methyl esters of fatty acids obtained under identical conditions. Peak areas were calculated with an analog electronic Packard Integrator apparatus.

\section{RESULTS}

\section{Isolation of 5'-IMP producing mutants}

The genealogy of the 5'-IMP producing mutants used in this work is presented in Fig. 1. Isolation and characterization of the two mutants, KY13102 and KY13171, were previously described by Furuya et al. ${ }^{11}$ and Kato et $a l .{ }^{3)}$ Both strains were adenine-leaky auxotrophic mutants whose growth was stimulated by adenine or adenosine.

In order to release the regulation in de novo purine nucleotide biosynthesis by guanine nu- 


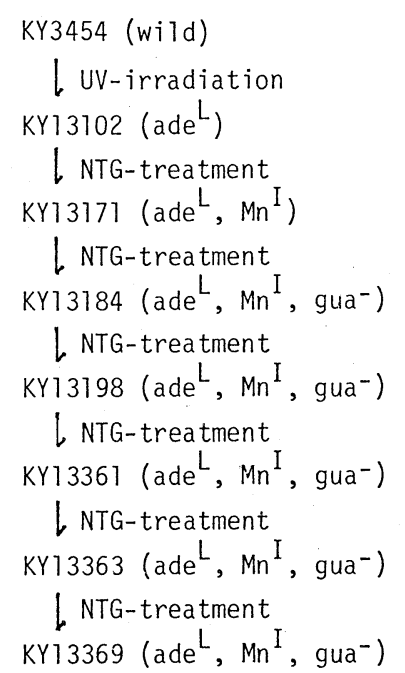

FIG. 1. Genealogy of B. ammoniagenes Mutants Producing 5'-IMP.

The following symbols are used: $\operatorname{ade}^{\mathrm{L}}$, adenine-leaky requirement; gua ${ }^{-}$, guanine requirement; $\mathrm{Mn}^{\mathrm{I}}, \mathrm{Mn}^{2+}$. insensitive; NTG, $N$-methyl- $N^{\prime}$-nitro- $N$-nitrosoguanidine.

cleotides, mutant KY13184 which is devoid of IMP dehydrogenase and requirement of guanine for growth, was induced by treatment of KY13171 with $N$-methyl- $N$ '-nitro- $N$-nitrosoguanidine (NTG). All further mutants were derived from KY13184 and obtained by repeated mutation and isolation. The 5'-IMP productivities of the mutants were tested under excessive $\mathrm{Mn}^{2+}$ conditions and KY13198, KY13361, KY13363 and KY13369 were selected as higher producers through each cycle of mutagenesis.

\section{Accumulation of 5'-IMP and Hx by mutants}

The accumulation of $5^{\prime}$-IMP and $\mathrm{Hx}$ in $250 \mathrm{ml}$ Erlenmeyer flasks by the series of 5'IMP producing mutants described above is shown in Table I. Under excessive $\mathrm{Mn}^{2+}$ conditions, an adenine-leaky auxotrophic mutant, KY13102, accumulated a large amount of $\mathrm{Hx}$ which is a degradation product of $5^{\prime}-$ IMP, and only a small amount of 5'-IMP. On the contrary, a Mn-insensitive mutant, KY13171, accumulated more 5'-IMP than $\mathrm{Hx}$ even in the presence of excess $\mathrm{Mn}^{2+}$. These results are fairly consistent with our earlier
Table I. ACCumulation OF 5'-IMP AND Hx By VARIOUS MUTANTS UNDER ExCESSIVE $\mathrm{Mn}^{2+}$ CONDITIONS

\begin{tabular}{cccc}
\hline Strain & $\begin{array}{c}5^{\prime}-\mathrm{IMP}^{a} \\
(\mathrm{mg} / \mathrm{ml})\end{array}$ & $\begin{array}{c}\mathrm{Hx}^{b} \\
(\mathrm{mg} / \mathrm{ml})\end{array}$ & $\begin{array}{r}\text { Total 5'-IMP } \\
(\mathrm{mg} / \mathrm{ml})\end{array}$ \\
\hline KY13102 & $1 \sim 2$ & $8 \sim 10$ & $9 \sim 12$ \\
KY13171 & $7 \sim 8$ & $4 \sim 6$ & $11 \sim 13$ \\
KY13184 & $8 \sim 10$ & $7 \sim 8$ & $15 \sim 18$ \\
KY13198 & $9 \sim 12$ & $5 \sim 6$ & $15 \sim 18$ \\
KY13361 & $12 \sim 16$ & $2 \sim 3$ & $15 \sim 19$ \\
KY13363 & $18 \sim 20$ & Trace & $18 \sim 20$ \\
KY13369 & $20 \sim 27$ & Trace & $20 \sim 27$ \\
\hline
\end{tabular}

a Expressed as 5'-IMP $\cdot \mathrm{Na}_{2} \cdot 7.5 \mathrm{H}_{2} \mathrm{O}$.

${ }^{b}$ Expressed as $5^{\prime}$-IMP $\cdot \mathrm{Na}_{2} \cdot 7.5 \mathrm{H}_{2} \mathrm{O}$ calculated from accumulated amounts of $\mathrm{Hx}$.

Sum of $a$ and $b$.

observations. ${ }^{1,3)}$

A mutant, KY13184, which was derived from KY13171 by addition of guanine auxotrophy, exhibited higher productivities of the 5'-IMP and Hx than the parent. KY13198, KY13361 and KY13363 exhibited a 10 to $20 \%$ increased level of 5'-IMP productivity, and concomitantly a decreased level of $\mathrm{Hx}$ productivity compared to each parent. Total 5'IMP, that is, the sum of $5^{\prime}$-IMP and $\mathrm{Hx}$ (calculated as $5^{\prime}$-IMP), produced by these mutants was almost the same as that produced by KY13184. This may imply that de novo biosynthetic rates of purine nucleotide did not change during successive mutagenic treatment in these three mutants.

\section{5'-IMP degrading activity}

The results described above suggest the following two possibilities: The increase of $5^{\prime}$ IMP accumulation on multi-stage mutagenesis is caused either by changes in 5'-IMP degrading activity or by alteration in cell membrane permeability for 5'-IMP. Therefore the 5'-IMP degrading activities in the cytoplasm and cell envelope were measured in some of these mutants exhibiting different productivity of 5'IMP. The cell envelope fraction was prepared according to the procedure of Spratt. ${ }^{10)}$ As shown in Table II, it was found that degrading activities were extremely weak, compared with those reported for various microorganisms ${ }^{11}$ ) 
Table II. 5'-IMP Degrading Activity and Alkaline Phosphatase ACTIVITY In VARIOUS MUTANTS

\begin{tabular}{|c|c|c|c|}
\hline \multirow{2}{*}{ Strain } & \multicolumn{2}{|c|}{$5^{\prime}$-IMP degrading activity ${ }^{a}$} & \multirow{2}{*}{$\begin{array}{c}\text { Alkaline phosphatase } \\
\text { activity }^{b}\end{array}$} \\
\hline & In cell extracts & In cell envelopes & \\
\hline KY13102 & 0.0386 & 0.239 & 3.17 \\
\hline KY13171 & 0.0245 & $\mathrm{ND}^{c}$ & 2.73 \\
\hline KY13184 & 0.0239 & 0.365 & 1.47 \\
\hline KY13198 & 0.0386 & ND & ND \\
\hline KY13363 & 0.0349 & ND & ND \\
\hline KY13369 & 0.0339 & 0.447 & 2.40 \\
\hline
\end{tabular}

a $\mu$ mol of degraded $5^{\prime}-\mathrm{IMP} / \mathrm{hr} / \mathrm{mg}$ protein.

b $\Delta \mathrm{OD}_{410} \mathrm{~nm} / \mathrm{min} / \mathrm{mg}$ protein.

$c$ ND, not determined.

and that no simple relationship was observed between 5'-IMP degrading activities in either the cytoplasm or cell envelope and 5'-IMP productivity. Similarly alkaline phosphatase activity seems unlikely to be correlated with the increased accumulation of 5'-IMP, though the three mutants exhibited slightly decreased levels of activity than a Mn-sensitive strain, KY13102 (Table II).

\section{Phospholipid content and composition}

From the results described above, it seems reasonable to consider that the increase of $5^{\prime}$ IMP accumulation on multi-stage mutagenesis was caused by alteration of the permeability barrier for 5'-IMP excretion. In order to confirm this, the components of the cell membrane of these mutants were analyzed.

The lipids of KY3454, a wild type strain of B. ammoniagenes and KY13363, a 5'-IMP high producing mutant, were extracted as described in Materials and Methods. The phospholipid content was calculated to be 66.7 to 78.4 and $84.3 \mu$ moles per gram dry cell weight, respectively. These data are fairly similar to those reported for various microorganisms. ${ }^{8)}$

The phospholipid components were separated and identified by comparing their $R f$ values with those of authentic standards in three solvent systems. In addition, the separated individual phospholipids were reacted with ninhydrin and acid molybdate (Dittmer's reagent) color reagents. Both strains had phosphatidylglycerol as the major component (more than $90 \%$ of total phospholipid), and phosphatidylethanolamine and cardiolipin as minor components (data not shown). In the end, it was concluded that there was no difference in phospholipid composition between the wild type strain and the 5'-IMP high producing mutant.

\section{Fatty acid composition}

It has been demonstrated that in KY13102, a Mn-sensitive mutant, $\mathrm{Mn}^{2+}$ affected the amounts of fatty acids in the cells resulting in the changes of membrane permeability for $5^{\prime}$ IMP. ${ }^{2)}$ Therefore it was interesting to study how the increased accumulation of $5^{\prime}$-IMP is related with the amounts and kinds of fatty acids in the mutants described here.

As shown in Fig. 2, two major peaks were detected at the 14th and 18th min and identified as palmitic acid and oleic acid, respectively. Minor peaks detected at the 10th and 17 th min were identified as myristic acid and stearic acid, respectively. Chromatograms of mutants other than KY13102 and KY13171 showed almost the same patterns as that of KY13171. Oleic acid content was calculated as the sum of stearic acid and oleic acid with an integrator apparatus, because stearic acid contents in all mutants were only a little and it was not well resolved from oleic acid (Fig. 2).

It is noteworthy that all $5^{\prime}$-IMP producing 


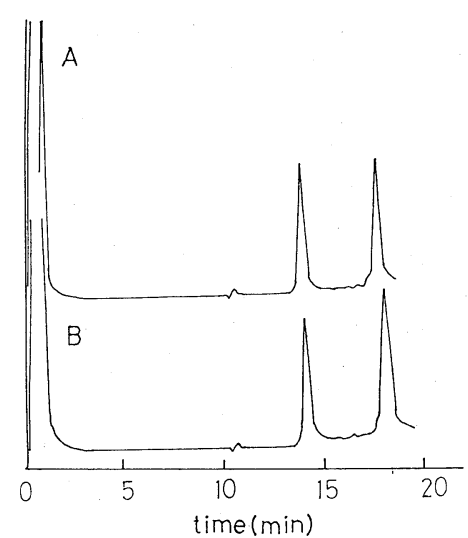

FIG. 2. Gas-liquid Chromatograms of Cellular Fatty Acids in KY13102 and KY13171.

Operation conditions were described in MATERIALS AND Methods. (A) KY13102; (B) KY13171.

Table III. Content of Cellular Fatty Acids in Wild Type Strain, KY3454 AND VARIOUS 5'-IMP Producing Mutants

\begin{tabular}{lcccc}
\hline & \multicolumn{3}{c}{ Fatty acid $(\%)$} & $\begin{array}{c}\text { Total FA } \\
\text { Strain }\end{array}$ \\
\cline { 2 - 4 } & & & & \\
& $\mathrm{C}_{14}$ & $\mathrm{C}_{16}$ & $\mathrm{C}_{18}+\mathrm{C}_{18}=$ & \\
\hline KY3454 & 4.2 & 55.3 & 39.5 & 20.25 \\
KY13102 & 1.2 & 51.6 & 46.9 & 31.64 \\
KY13171 & 1.0 & 44.4 & 54.5 & 37.32 \\
KY13184 & 0.6 & 48.1 & 51.3 & 32.63 \\
KY13198 & 0.4 & 40.0 & 59.6 & 33.98 \\
KY13363 & 0.6 & 39.6 & 59.8 & 35.81 \\
KY13369 & 0.7 & 43.5 & 55.7 & 34.53 \\
\hline
\end{tabular}

a FA, fatty acid.

b DCW, dry cell weight.

mutants contained about 1.5 times more total fatty acids than the wild type strain, KY3454 , and that myristic acid contents of the 5'-IMP producers were extremely low (less than $20 \%$ of KY3454). As shown in Table III, these changes appear to originate in the adenine-leaky auxotroph (KY13102) and not to correlate closely with $5^{\prime}$-IMP productivity. The most interesting result is that the palmitic acid content of a Mn-insensitive mutant, KY13171, decreased remarkably with a concomitant increase of oleic acid in comparison with a Mn-sensitive mutant, KY13102, indicating that quantitative alter- ation of fatty acid components may be correlated with Mn-insensitivity in accumulation of 5'-IMP. Among Mn-insensitive mutants (KY13171 to KY13369 in Table III), a relationship between fatty acid composition and 5'-IMP productivity was not observed. For instance, KY13363 exhibited 1.5 times more 5'-IMP productivity than KY13198, although the contents and composition of fatty acids in both mutants were almost the same.

\section{DISCUSSION}

In this report, we described that strain improvement for 5'-IMP productivity was achieved through repeated cycles of mutation and isolation (Fig. 1). One of the mutants, KY13369, accumulated about 27 grams of 5'IMP per liter at the maximum in shaking flasks and this corresponds to more than a $20 \%$ yield of 5'-IMP based on consumed sugar.

It has been pointed out that the following three factors were required for effective production of 5'-IMP-1) release from feedback regulation working in de novo purine nucleotide biosynthesis, 2) lack of 5'-IMP degrading activities and 3) removal of the membrane permeability barrier for 5'-IMP excretion. ${ }^{12)}$ Therefore it is interesting to determine which factor mainly contributes to the increased accumulation of $5^{\prime}$-IMP.

Alteration in de novo purine nucleotide biosynthesis: Kotani et al. $^{13)}$ reported that the accumulation of inosine was markedly improved by introducing guanine requirement to an inosine producing strain of $B$. ammoniagenes.

A similar approach was taken in this work and a mutant, KY13184, was derived from KY13171 to which the guanine requiring character was added. Although KY13184 exhibited higher productivities of both $5^{\prime}$-IMP and $\mathrm{Hx}$ than the parent strain, the increase of $5^{\prime}$-IMP productivity was not so drastic as observed in the inosine accumulation. This discrepancy seems to suggest that other factors are more important for a 5'-IMP producer than the release from the feedback regulation system. It 
is, however, noteworthy that productivity of revertants of the guanine auxotroph was drastically reduced (data not shown).

All further improved mutants derived from KY13184 through repeated mutagenesis showed increased productivity of 5'-IMP with a concomitant decrease of $\mathrm{Hx}$ productivity. The fact that the sum of $5^{\prime}$-IMP and $\mathrm{Hx}$, that is, total 5'-IMP, remained constant through KY13184 to KY13363, indicates no alteration in de novo purine nucleotide biosynthetic activity. Whether the increased productivity of KY13369 is caused by the alteration in de novo purine nucleotide biosynthesis or other factors is unclear.

Alteration in 5'-IMP degrading activity: Lack of nucleotide degrading activity is considered to be one of the required factors for nucleotide production. In fact, Takayama et $a l{ }^{14)}$ succeeded in improvement of salvage synthesis of 5 -IMP or $5^{\prime}$-guanylic acid from $\mathrm{Hx}$ or guanine, respectively, using B. ammoniagenes mutants whose 5'-nucleotide degrading activities were considerably reduced. Similarly Akiya et al. ${ }^{15)}$ isolated mutants of Bacillus subtilis whose nucleotide degrading activities were markedly reduced and succeeded in accumulation of about 3.4 grams of 5'-IMP per liter.

As mentioned above, the productivity of $\mathrm{Hx}$ of the improved mutants derived from KY13184 decreased concomitant with the increase in 5'-IMP accumulation. Therefore it is of interest to determine whether alteration in 5'-IMP degrading activity could be due to this increased accumulation of 5'-IMP. As shown in Table II, no simple relationship was observed between the productivity and 5'-IMP degrading activities or alkaline phosphatase activity. These results seem to suggest that the degree of degradation of $5^{\prime}$-IMP is not determined merely by 5'-IMP degrading activity but by the balance of 5'-IMP degrading activity and membrane permeability for $5^{\prime}$ IMP.

Alteration in membrane permeability for $5^{\prime}$ IMP: From the results described above, it seems reasonable to consider that the increase of 5'-IMP accumulation of the improved mutants derived from KY13184 may be due to improved membrane permeability for 5'-IMP.

The importance of membrane permeability for efficient accumulation of metabolites has been noted in the industrial fermentation field. A typical example has been shown in the fermentative production of glutamic acid. ${ }^{8}$ Improvement of membrane permeability is considered to be an effective factor for increasing the yield of 5'-IMP as in the case of glutamic acid fermentation, since bacterial cytoplasmic membranes are generally impermeable for nucleotides. In intensive work on the mechanisms 5'-IMP accumulation by $B$. ammoniagenes mutant KY13102, it was demonstrated that limitation of $\mathrm{Mn}^{2+}$ leads to alteration in the contents of cellular fatty acids, resulting in the elimination of the permeability barrier for $5^{\prime}$ IMP. ${ }^{2)}$ Therefore it is worth determining how alteration in components of cell membrane is correlated with productivities of 5'-IMP of the improved mutants.

Comparison of contents and composition of phospholipids between a wild type strain, KY3454, and a 5'-IMP high producing mutant, KY13363, indicated that both strains contained only phosphatidylglycerol as a major component and that the latter contained slightly more phospholipids than the former.

Furthermore, we determined the contents and composition of cellular fatty acids in the improved mutants with various $5^{\prime}$-IMP productivities. Contents of oleic acid in all Mninsensitive mutants, KY13171 to KY13369, reached more than $50 \%$ of total fatty acids, while that in a Mn-sensitive mutant, KY13102, less than $50 \%$ of total fatty acids. This difference in oleic acid content could partly explain the changes of sensitivity to $\mathrm{Mn}^{2+}$ in accumulation of $5^{\prime}$-IMP. The most interesting fact is that all $5^{\prime}$-IMP producing mutants contained 1.5 times more total fatty acids than the wild type strain, KY3454. Takinami et al. ${ }^{8)}$ have demonstrated that impairment of phospholipid biosynthesis reduced the amounts of phospholipids in the cell membrane, resulting in the 
elimination of the permeability barrier for glutamic acid. This clearly indicates that excretion mechanisms of $5^{\prime}$-IMP in the permeability mutants are different from those of glutamic acid. More detailed work on the excretion mechanisms of 5'-IMP should be carried out in the near future.

\section{REFERENCES}

1) A. Furuya, S. Abe and S. Kinoshita, Appl. Microbiol., 16, 981 (1969).

2) A. Furuya, S. Abe and S. Kinoshita, Agric. Biol. Chem., 34, 210 (1970).

3) F. Kato, A. Furuya and S. Abe, Agric. Biol. Chem., 35, 1061 (1971).

4) T. Nara, T. Komuro, M. Misawa and S. Kinoshita, Agric. Biol. Chem., 33, 739 (1969).
5) A. Furuya, M. Misawa and S. Kinoshita, Agric. Biol. Chem., 31, 1351 (1969).

6) I. Shiio and H. Ozaki, J. Biochem., 83, 409 (1978).

7) P. S. Chen Jr., T. Toribara and H. Waner, Anal. Chem., 28, 1756 (1956).

8) K. Takinami, H. Yoshii, Y. Yamada, H. Okada and K. Kinoshita, Amino Acid and Nucleic Acid, 18, 120 (1968)

9) F. E. Luddy, R. A. Barford, R. W. Riemenshneider, J. Am. Oil Soc., 37, 447 (1960).

10) B. G. Spratt, Eur. J. Biochem., 72, 341 (1977).

11) M. Misawa, T. Nara and K. Nakayama, Nippon Nôgeikagaku Kaishi, 38, 100 (1967).

12) A. Furuya, Hakko Kyokaishi, 36, 1036 (1978).

13) K. Kotani, K. Yamaguchi, F. Kato and A. Furuya, Agric. Biol. Chem., 42, 399 (1978).

14) K. Takayama, A. Furuya and S. Abe, Amino Acid and Nucleic Acid, 22, 15 (1970).

15) T. Akiya, Y. Midorikawa, A. Kuninaka, H. Yoshida and Y. Ikeda, Agric. Biol. Chem., 36, 277 (1972). 